\title{
Descifrando el papel del entrenamiento interválico de alta intensidad en el cáncer de mama: revisión sistemática \\ Deciphering the role of high-intensity interval training in breast cancer: systematic review \\ Cristian González García \\ Universidad de Murcia (España)
}

Resumen. El cáncer de mama es uno de los más extendidos y proliferativo a nivel mundial. Esto provoca un gran impacto en el gasto sanitario. Aunque se sabe que el ejercicio físico mejora diferentes aspectos relacionados con el cáncer, hoy en día se desconoce qué tipo de entrenamiento o intensidad es mejor para esto. El propósito de esta revisión sistemática fue conocer los efectos fisiológicos, cognitivos y económicos que aporta el HIIT a este tipo de pacientes. La búsqueda se realizó en las bases de datos PubMed, Web of Science, MEDLINE y Scopus, a partir de los descriptores «cancer, high intensity interval training y breast». De los 78 obtenidos inicialmente, 32 cumplieron todos los criterios de inclusión. Los estudios analizados se centran principalmente en añadir un programa HIIT al tratamiento de personas que padecen, han padecido o tiene algo riesgo de padecerlo viendo efectos positivos a nivel de capacidades físicas, cognitivo y financiero. Este método es seguro y factible, y conlleva un aumento de la calidad de vida de estos pacientes. Además, puede mejorar las capacidades físicas, su calidad de vida, se reducen las tasas de finalización del tratamiento, hospitalización y gasto sanitario. Estos efectos beneficiosos deben ser tenidos en cuenta por los profesionales del ámbito médico, fisioterapeutas y profesionales en el deporte en cuanto a su implementación en estos pacientes.

Palabras clave: cáncer, entrenamiento interválico de alta intensidad, mama.

Summary. Breast cancer is one of the most widespread and proliferative cancers worldwide. This has a great impact on healthcare spending. Although it is known that physical exercise improves different aspects related to cancer, it is currently unknown what type of training or intensity is best for this. The prupose of this systematic review was to find out the physiological, cognitive and economic effects of HIIT for this type of patient. The search was carried out in the databases PubMed, Web of Science, MEDLINE and Scopus, using the descriptors «cancer, high intensity interval training and breast». Of the 78 initially obtained, 32 met all the inclusion criteria. The studies analysed focus mainly on adding a HIIT programme to the treatment of people who suffer, have suffered or are at risk of suffering from cancer, seeing the positive effects on physical, cognitive and financial capacities. This method is safe and feasible, and leads to an increase in the quality of life of these patients. In addition, it can improve physical abilities and quality of life, reduce treatment completion rates, hospitalization and healthcare costs. Its implementation in cancer patients should be taken into account by professionals in the medical field, as well as physiotherapists and sports professionals, given its benefits.

Keywords: cancer, high intensity interval training, breast.

\section{Introducción}

El cáncer es el nombre que se le da a un grupo de enfermedades relacionadas y que puede afectar a diversas partes del cuerpo. Según la Organización Mundial de la Salud (OMS), en 2018, se consideraba la segunda causa de muerte en el mundo, siendo un $17 \%$ de las defunciones a nivel mundial. Estas se producen en su mayoría en países de ingresos medios o bajos. Entre los factores de riesgo que ocasionan la muerte por cáncer;

Fecha recepción: 24-07-21. Fecha de aceptación: 16-10-21

Cristian González García

cristiangg97@gmail.com se encuentran el tabaquismo (Forouzanfar et al., 2016), infecciones por virus oncogénicas (Plummer et al., 2016) y factores conductuales y dietéticos. Por lo que el impacto económico, según la Asociación Española Contra el Cáncer (AECC), que ocasiona esta enfermedad es alto a nivel sanitario, específicamente 19300 millones de euros.

La metástasis es la primera causa de muerte por cáncer, consiste en la proliferación excesiva de células anómalas de una forma rápida y que pueden invadir cualquier parte del cuerpo, incluido los órganos (OMS, 2018). Factores como el tabaquismo aumenta la probabilidad de que la metástasis aparezca o vuelva a aparecer (Foerster et al., 2018).

Si diferenciamos entre hombres y mujeres, basán- 
donos en las estadísticas de la Agencia Internacional para la Investigación del Cáncer (IARC) (2020), el cáncer de mama es el más extendido a nivel mundial sobre todo en Norte América, Europa, Asia y el norte de África. La mayor tasa de incidencia de cáncer de mama se da en Europa, sobre todo en Francia, Bélgica e Italia (Fig. 1).

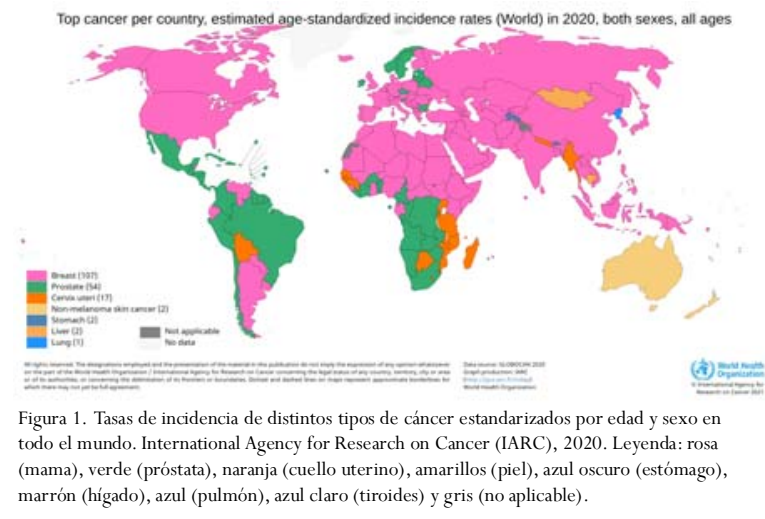

En 2020, se dieron más de 18 millones de casos de cáncer en todo el mundo, teniendo en cuenta a ambos sexos y a todas las edades. El mayor número de casos nuevos apareció en cáncer de pulmón $(11,4 \%)$, seguido de cáncer de mama $(11,7 \%)$, colorrectal $(10 \%)$ y próstata $(7,3 \%)$ (Fig. 2).
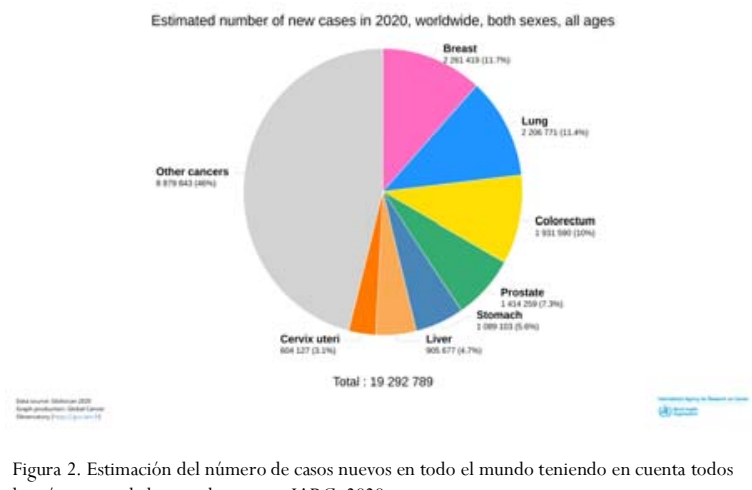

los cánceres, edades y ambos sexos. IARC, 2020

La Agencia Internacional para la Investigación en Cáncer (IARC) prevé un aumento de esta enfermedad en un 38,5\% entre 2018 y 2040. Además, se estima que

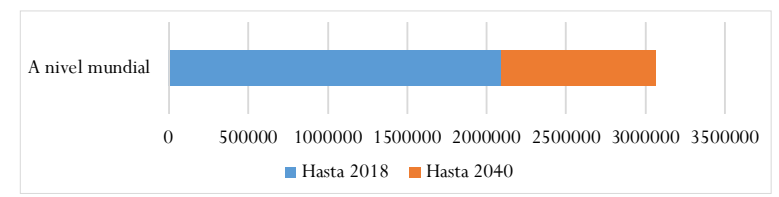

Figura 3. Estimación del número de casos nuevos de cáncer de mama del 2018 al 2040, teniendo en cuenta todas las edades. IARC, 2018

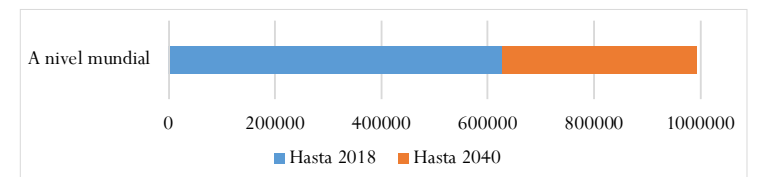

Figura 4. Estimación del número de muertes del 2018 al 2040 en personas con cáncer de mama de todas las edades. IARC, 2018. la mortalidad por cáncer aumentará en un 41\%. En 2018, sólo el 47\% sobrevivía a esta enfermedad, aunque se prevé que esta cifra disminuya (44\%) en 2040 (IARC, 2018) (Fig. 3).

Como hemos comentado anteriormente, el cáncer de mama es uno de los que más proliferan y más se extiende por todo el mundo. Según la IARC (2018), el número de casos va aumentar hasta el 2040 de 2088 849 casos a 3500 000, esto supone un aumento del 27,75\% (Fig. 3). Dentro de esto, la mortalidad por cáncer de mama va a aumentar un 36,5\% respecto a 2018 (Fig. 4).

Atendiendo a las edades (Fig. 5), la probabilidad de desarrollar cáncer en España en 2019 empieza a aumentar a partir de los 40 años. Teniendo en cuenta que a partir de los 65 años se produce el mayor auge de probabilidad de padecerlo, desde esta edad en adelante se produce una diferencia significativa entre hombres y mujeres, llegando a darse hasta un 20\% de probabilidad más en hombres que en mujeres.

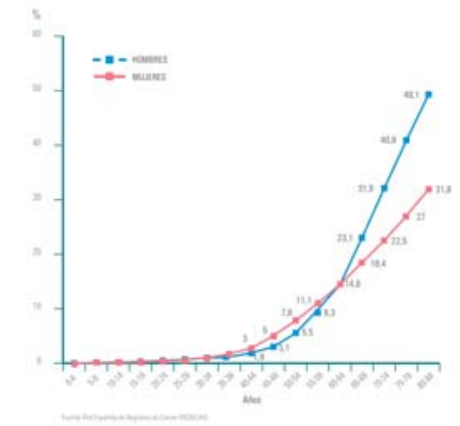

Figura 5. Probabilidad de desarrollar cáncer en España en 2019. Sociedad Española de Oncología Médica (SEOM), 2019.

Tras ver estas estadísticas, uno de los tipos de cáncer más preocupantes por expansión geográfica y proliferación de casos es el de mama. Por lo tanto, este es el cáncer que se va abordar durante nuestra revisión bibliográfica.

En este trabajo queríamos determinar la evidencia científica relacionada con los beneficios de la práctica de ejercicio en pacientes con cáncer, tanto en el periodo de tratamiento oncológico como después de este.

El ejercicio físico puede ser muy beneficioso en aspectos como la salud cardiovascular, la fatiga, la fuerza muscular, la calidad de vida y la depresión. Por lo tanto, el ejercicio ayuda a controlar tanto el estado de salud mental y física y, con esto, la calidad de vida de los pacientes que padecen cáncer (Fuller et al., 2018).

Un punto muy importante en el que afecta el ejercicio físico es en paliar o reducir los efectos secundarios que produce la medicación (D’Ascenzi et al., 2019; 
Fuller et al., 2018).

Según la revisión sistemática realizada por Zhang et al. (2019), los ejercicios de resistencia y ejercicios aeróbicos tienen efectos beneficiosos y son seguros para los pacientes con cáncer aportando una mayor calidad de vida. Pero los ejercicios combinados, compuestos por ejercicios aeróbicos y entrenamiento de resistencia, son más efectivos y seguros para la mejora de calidad de vida de estos pacientes.

Sin embargo, no todas las intensidades tienen el mismo efecto en personas con cáncer. Según Dennett et al. (2016), el ejercicio aeróbico a intensidad moderada reduce la fatiga y mejora la calidad de vida de estas persona respecto al ejercicio aeróbico de baja intensidad. Además, según Karlsen et al. (2017) los entrenamientos interválicos de alta intensidad (HIIT) tienen mayores resultados que los entrenamientos continuos a intensidad moderada.

El HIIT se ha extendido por la población como una actividad recreativa por sus beneficios en la condición física y por realizarse en sesiones de corta duración. Existen varios estudios que demuestran estos beneficios tanto en personas jóvenes como en adultos sanos (Emberts et al., 2013; Gibala \& McGee, 2008; Pérez \& Chaves, 2015). Este tipo de entrenamiento consiste en intervalos breves a una intensidad máxima, o cercana a la máxima, con descansos de pocos segundos en función de la duración de la alta intensidad o con ejercicios de baja intensidad en los descansos (Gibala \& McGee, 2008).

En el HIIT se produce una mayor motivación en personas adultas activas sanas y sedentarias, dando una mayor adherencia a estas personas para seguir realizando ejercicio físico. Esto puede deberse al poco tiempo que se necesita, ya que el volumen es muy bajo (Heinrich et al., 2014; Wilke et al., 2019). Incluso las tasas de abandono respecto al entrenamiento convencional son mucho menores en personas sedentarias (Reljic et al., 2019), por lo que en personas con cáncer de mama que no hayan realizado anteriormente ejercicio físico puede que tengan un mayor adherencia. Podemos observar en el estudio de García et al. (2019), que este tipo de entrenamiento puede ser una buena herramienta para personas con Diabetes Mellitus tipo 2. Además, puede mejorar la presión arterial y el comportamiento de la frecuencia cardiaca en mujeres sanas (Neto et al., 2022). En el ámbito escolar, las niñas también tienen beneficios cuando usan el HIIT, reduciendo su índice de masa corporal y mejorando su condición física (Grisalez et al., 2021; Segovia \& Gutiérrez, 2020). El HIIT puede ser una opción para añadir como intervención en la salud pública (Heinrich et al., 2014).

Si tenemos en cuenta la edad del paciente, podemos pensar que el HIIT no es adecuado para personas mayores de 60 años, pero según la revisión de Gomez-Piqueras \& Sanchez-Gonzalez (2019) este tipo de entrenamiento es válido y seguro teniendo beneficios cardiovasculares, pulmonares, hemodinámicos, lipídicos, musculares y cognitivos.

Tras todos estos datos expuestos anteriormente, tenemos la hipótesis de que los resultados se incrementan cuando se realiza el entrenamiento de forma interválica, es posible que añadir entrenamientos interválicos de alta intensidad (HIIT) al tratamiento podría mejorar factores como las capacidades físicas, fisiológicas y calidad de vida, que afectan al paciente con cáncer.

\section{Objetivo del trabajo}

El propósito general de esta revisión sistemática es conocer los efectos de los programas que añaden entrenamiento interválico de alta intensidad en personas con cáncer de mama o que lo han superado. Específicamente los efectos a nivel fisiológico, cognitivo y económico.

\section{Material y métodos}

\section{Tipo de estudio}

Revisión sistemática de la literatura. La metodología se basa en la búsqueda en bases de datos establecidas, lectura crítica de la literatura, síntesis de resultados y evaluación de los mismos.

\section{Búsqueda bibliográfica}

Para alcanzar los objetivos propuestos se realizó una búsqueda en las siguientes bases de datos: PubMed, Web of Science, Medline y Scopus, que se encuentran entre las más importante en el ámbito de la salud y la actividad física. Se examinaron todos los estudios realizados desde enero de 2016 hasta agosto de 2020, en inglés y español para establecer la base de este análisis. Las palabras para la búsqueda en inglés, fueron «cancer», unida por la conjunción «AND» a las palabras clave, «high intensity interval training» $\mathrm{y}$ «breast» (descriptores DeCS y MeSH).

\section{Criterios de inclusión / exclusión}

Se establecieron los siguientes criterios de inclusión: -Artículos originales.

-Idioma: trabajos redactados en inglés o español.

-Estudios con un programa de intervención HIIT. 
-Estudios con muestra que padezca o haya padecido cáncer de mama.

Se establecieron los siguientes criterios de exclusión:

-Revisiones sistemáticas.

-Idioma: trabajos no redactados en español o inglés.

-Estudios que no aplicaban un programa de intervención HIIT.

-Estudios con muestra que no padezca o haya padecido cáncer de mama.

\section{Selección de estudios}

Tras la identificación y selección de los artículos, se realizó una evaluación completa teniendo en cuenta los criterios de elegibilidad iniciales. Se utilizaron los siguientes criterios de inclusión: que fueran artículos originales, estudios en los que se hubiesen llevado a cabo un programa interválico de alta intensidad en la intervención en personas que padezcan cáncer o que lo hayan superado y que fuesen redactados en inglés o español. Por lo tanto, se excluyeron del análisis las revisiones sistemáticas, todos aquellos artículos que no estuvieran escritos en español o inglés, los que no aplicaban HIIT como programa de intervención y aquellos en los que los participantes no padezcan o hayan padecido cáncer.

\section{Resultados}

Obtuvimos el siguiente diagrama de flujo para la búsqueda centrada en cáncer de mama (Fig. 6). En la Tabla 1 se muestran los artículos seleccionados para realizar la revisión sistemática entre un programa con intervención HIIT y cáncer de mama, aportando resultados sobre las capacidades físicas relacionadas con la salud (resistencia, fuerza muscular, composición corporal y fatiga), aspectos fisiológicos, calidad de vida (efectos psicológicos, dolor, síntomas y efectos secundarios), seguridad y factibilidad del programa y tasas de finalización, hospitalización y costos sanitarios.

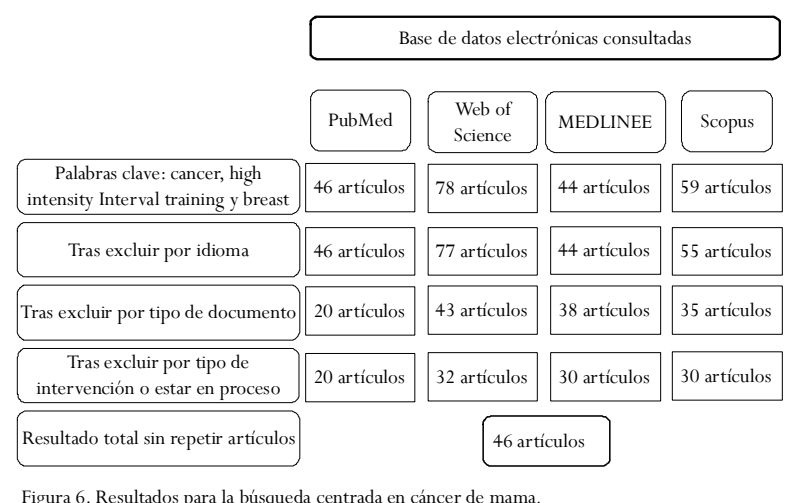

\section{Discusión}

El propósito de esta revisión, es conocer los efectos y beneficios del método HIIT en el tratamiento de personas con cáncer de mama, sobre la mejora de sus capacidades físicas relacionadas con la salud (resistencia, fuerza muscular, composición corporal y fatiga), aspectos fisiológicos, calidad de vida (efectos psicológicos, dolor, síntomas y efectos secundarios), seguridad y factibilidad del programa y tasas de finalización, hospitalización y costos sanitarios, desde 2016 a 2020.

\section{Capacidades físicas}

En personas con cáncer de mama existe un aumento significativo de la cantidad máxima de oxígeno que el organismo puede absorber ( $\mathrm{V}^{2}$ máx.) (A. M. Alizadeh et al., 2019; Ansund et al., 2021), manteniendo o evitando la aptitud cardiorrespiratoria en pacientes con un tratamiento de antraciclina (Lee, Kang, Mack, Mortimer, Sattler, Salem, \& Dieli-Conwright, 2019; Lee et al., 2020; Mijwel, Backman, Bolam, Olofsson, et al., 2018), en pacientes obesos con esta enfermedad (Schulz et al., 2018).

En personas supervivientes del cáncer de mama dentro del periodo de rehabilitación asegura una mayor resistencia (Midtgaard et al., 2013; Schmitt et al., 2016; Toohey et al., 2020) al igual que las personas con alto riesgo de padecer cáncer de mama (Coletta et al., 2019). Mejorando la regulación cardiaca en mujeres supervivientes (Toohey et al., 2020) e incluso previniendo el deterioro de la función cardiaca en mujeres con cáncer de mama sometidas a quimioterapia (Chung et al., 2021).

La fuerza muscular se ve aumentada significativamente tanto en los miembros superiores como inferiores, en pacientes con cáncer de mama con un tratamiento basado en quimioterapia (Adamsen et al., 2009; Midtgaard et al., 2013; Mijwel, Jervaeus, et al., 2019; Pagola et al., 2020), tanto en personas que habían pasado por cirugía como las que no (Mijwel, Backman, Bolam, Olofsson, et al., 2018) e incluso con una intervención de 6 semanas se puede obtener un aumento del $26 \%$ de fuerza como promedio (Schulz et al., 2018). Además, el aumento de fuerza en las extremidades inferiores sigue siendo significativo a los 2 años de intervención (Bolam et al., 2019).

La fatiga relacionada con el cáncer de mama en personas con tratamiento de quimioterapia se ve contrarrestada (Bolam et al., 2019; Hiensch et al., 2021; Mijwel, Backman, Bolam, Jervaeus, et al., 2018) y se 
Tabla 1.

\begin{tabular}{|c|c|c|c|c|c|}
\hline $\begin{array}{l}\text { Autores } \\
\text { / Año de } \\
\text { publicación }\end{array}$ & Revista & Muestra & Objetivo & $\begin{array}{l}\text { Duración } \\
\text { (intervención) }\end{array}$ & Conclusión \\
\hline $\begin{array}{l}\text { A. M. } \\
\text { Alizadeh } \\
\text { et al. (2019) }\end{array}$ & $\begin{array}{l}\text { Journal of Cancer } \\
\text { Research and Clinical } \\
\text { Oncology }\end{array}$ & $\begin{array}{l}\mathrm{n}=52 \\
\mathrm{GC}=26 \\
\mathrm{GH}=26 \\
31-69 \text { años }\end{array}$ & $\begin{array}{l}\text { Comprobar la hipótesis de que el entrenamiento de alta intensidad en } \\
\text { pacientes con cáncer de mama durante la terapia hormonal mejora el } \\
\text { estado de inflamación. }\end{array}$ & 12 semanas & $\begin{array}{l}\text { Efecto positivo del HIIT en la aptitud cardiorrespiratoria y citocinas } \\
\text { inflamatorias en mujeres con cáncer de mama en terapia hormonal. }\end{array}$ \\
\hline $\begin{array}{l}\text { S. Alizadeh } \\
\text { et al. (2019) }\end{array}$ & $\begin{array}{l}\text { Annals of Physical and } \\
\text { Rehabilitation } \\
\text { Medicine }\end{array}$ & $\begin{array}{l}\mathrm{n}=82 \\
\mathrm{GC}=15 \\
\mathrm{GCH}=15 \\
\mathrm{GH}=26 \\
\mathrm{GHH}=26 \\
\mathrm{GH}=48.42 \pm 7.54 \text { años } \\
\mathrm{GHH}=49.2 \pm 9.7 \text { años }\end{array}$ & $\begin{array}{l}\text { Determinar si el uso de microARN junto a HIIT es útil para las } \\
\text { decisiones terapéuticas. }\end{array}$ & 12 semanas & $\begin{array}{l}\text { El HIIT podría ayudar a suprimir los tumores respecto a la terapia hormonal } \\
\text { únicamente, los niveles séricos de miRs asociados al cáncer de mama } \\
\text { podrían ser buenos biomarcadores. }\end{array}$ \\
\hline $\begin{array}{l}\text { Blackwell } \\
\text { et al. (2020) }\end{array}$ & $\begin{array}{l}\text { Prostate Cancer and } \\
\text { Prostatic Diseases }\end{array}$ & $\begin{array}{l}\mathrm{N}=34 \\
72 \text { años }\end{array}$ & $\begin{array}{l}\text { Evaluar la eficacia del HIIT en la mejora de la aptitud } \\
\text { cardiorrespiratoria en personas que van a ser operadas de cáncer, con } \\
\text { una antelación de } 4 \text { semanas. }\end{array}$ & 4 semanas & $\begin{array}{l}\text { HIIT puede mejorar CRF y la salud cardiovascular, lo que representa } \\
\text { mejoras preoperatorias clínicamente significativas y alcanzables. }\end{array}$ \\
\hline $\begin{array}{l}\text { Bolam et al. } \\
(2019)\end{array}$ & $\begin{array}{l}\text { Breast Cancer Research } \\
\text { and Treatment }\end{array}$ & $\begin{array}{l}n=206 \\
18-70 \text { años }\end{array}$ & $\begin{array}{l}\text { Diferencias relacionadas con la salud y la AF entre dos grupos y grupo } \\
\text { control, y sus efectos dos años después. }\end{array}$ & 16 semanas & $\begin{array}{l}\text { La introducción del HIIT en el programa de entrenamiento fue positivo para } \\
\text { la fatiga asociada al cáncer, sus síntomas y la fuerza muscular respecto al } \\
\text { tratamiento habitual. Por lo que pueden darse beneficios a largo plazo. }\end{array}$ \\
\hline $\begin{array}{l}\text { Coletta et al. } \\
\text { (2019) }\end{array}$ & $\begin{array}{l}\text { Medicine \& Science in } \\
\text { Sports \& Exercise }\end{array}$ & $\begin{array}{l}\mathrm{n}=44 \\
\mathrm{GC}=14 \\
\mathrm{GIM}=14 \\
\mathrm{GH}=16 \\
\mathrm{GC}=63.35 \pm 7.0 \text { años } \\
\mathrm{GIM}=64.62 \pm 12.2 \text { años } \\
\mathrm{GH}=63.73 \pm 6.9 \text { años }\end{array}$ & $\begin{array}{l}\text { Demostrar la viabilidad del entrenamiento HIIT en mujer que padecen } \\
\text { sobrepeso u obesidad, riesgo de cáncer de mama y son } \\
\text { posmenopáusicas, así como su efecto cardiorrespiratorio y en su } \\
\text { composición corporal respecto a la intensidad moderada continua o la } \\
\text { atención común. }\end{array}$ & 12 semanas & $\begin{array}{l}\text { El GH es factible, seguro y parece promover mayores mejoras en CRF en } \\
\text { comparación con GIM y GC en mujeres con alto riesgo de cáncer de mama. }\end{array}$ \\
\hline $\begin{array}{l}\text { Lee et al. } \\
(2019)\end{array}$ & $\begin{array}{l}\text { Breast Cancer Research } \\
\text { and Treatment }\end{array}$ & $\begin{array}{l}\mathrm{N}=30 \\
\mathrm{GC}=15 \\
\mathrm{GH}=15 \\
46,9 \pm 9,8 \text { años }\end{array}$ & $\begin{array}{l}\text { Determinar los efectos de una intervención HIIT en la función } \\
\text { endotelial vascular y el grosor de la pared vascular en mujeres con } \\
\text { cáncer de mama sometidas a quimioterapia con antraciclinas. }\end{array}$ & 8 semanas & $\begin{array}{l}\text { HIIT mejoró la función endotelial vascular y mantuvo el grosor de la pared } \\
\text { en pacientes con cáncer de mama con un tratamiento de antraciclinas. }\end{array}$ \\
\hline $\begin{array}{l}\text { Lee et al. } \\
\text { (2019) }\end{array}$ & BMC Cancer & $\begin{array}{l}\mathrm{N}=30 \\
\mathrm{GC}=15 \\
\mathrm{GH}=15 \\
46,9 \pm 9,8 \text { años }\end{array}$ & $\begin{array}{l}\text { Determinar si una intervención HIIT mantiene el VO2max en mujeres } \\
\text { con cáncer de mama sometidas a quimioterapia con antraciclinas. }\end{array}$ & 8 semanas & $\begin{array}{l}\text { HIIT es una intervención de ejercicio factible para mantener la cantidad } \\
\text { máxima de oxígeno que el organismo puede absorber ( } \mathrm{VO}_{2} \text { máx) en } \\
\text { pacientes con cáncer de mama que reciben quimioterapia basada en } \\
\text { antraciclina. }\end{array}$ \\
\hline $\begin{array}{l}\text { Mijwel et al. } \\
\text { (2018) }\end{array}$ & $\begin{array}{l}\text { Breast Cancer Researcl } \\
\text { and Treatment }\end{array}$ & $\begin{array}{l}\mathrm{N}=240 \\
\mathrm{GC}=80 \\
\mathrm{RT}-\mathrm{HIIT}=80 \\
\text { AT-HIIT }=80 \\
18-70 \text { años }\end{array}$ & $\begin{array}{l}\text { Efectos de una intervención de resistencia y HIIT sobre la fatiga y } \\
\text { síntomas en personas con cáncer de mama que se encuentran en } \\
\text { quimioterapia. }\end{array}$ & 16 semanas & $\begin{array}{l}\text { Tanto la resistencia como el HIIT fueron eficaces para prevenir aumentos en } \\
\text { la CRF y para reducir los síntomas en pacientes durante la quimioterapia en } \\
\text { pacientes con cáncer de mama. HIIT, como un componente vital de la } \\
\text { rehabilitación del cáncer. }\end{array}$ \\
\hline $\begin{array}{l}\text { Mijwel et al. } \\
\text { (2018) }\end{array}$ & $\begin{array}{l}\text { Breast Cancer Research } \\
\text { and Treatment }\end{array}$ & $\begin{array}{l}\mathrm{N}=240 \\
\mathrm{GC}=80 \\
\mathrm{RT}-\mathrm{HIIT}=80 \\
\text { AT-HIIT }=80 \\
18-70 \text { años }\end{array}$ & $\begin{array}{l}\text { Efectos de una intervención combinada de resistencia y HIIT sobre el } \\
\text { dolor y resultados fisiológicos en personas con cáncer de mama que se } \\
\text { encuentran en quimioterapia. }\end{array}$ & 16 semanas & $\begin{array}{l}\text { El entrenamiento concurrente con HIIT mejoró significativamente la fuerza } \\
\text { muscular y redujeron la sensibilidad al dolor. Ambos programas de ejercicio } \\
\text { fueron bien tolerados y con una alta eficiencia en la prevención de aumentos } \\
\text { en la masa corporal y para prevenir la disminución de la resistencia } \\
\text { cardiorrespiratoria. }\end{array}$ \\
\hline $\begin{array}{l}\text { Mijwel et al. } \\
\text { (2020) }\end{array}$ & The Oncologist & $\begin{array}{l}\mathrm{N}=240 \\
\mathrm{GC}=80 \\
\mathrm{RT}-\mathrm{HIIT}=80 \\
\text { AT-HIIT }=80 \\
18-70 \text { años }\end{array}$ & $\begin{array}{l}\text { Efectos de una intervención combinada de resistencia y HIIT sobre la } \\
\text { finalización de la quimioterapia y hospitalización, así como las } \\
\text { concentraciones de células sanguineas en personas con cáncer de mama } \\
\text { que se encuentran en quimioterapia. }\end{array}$ & 16 semanas & $\begin{array}{l}\text { No se encontraron efectos beneficiosos con resistencia y HIIT sobre las tasas } \\
\text { de finalización de la quimioterapia. Sin embargo, el entrenamiento } \\
\text { combinado de resistencia y HIIT fueron efectivos para reducir las tasas de } \\
\text { hospitalización, y ambos grupos de ejercicio tuvieron un efecto positivo } \\
\text { sobre la trombocitopenia. }\end{array}$ \\
\hline $\begin{array}{l}\text { Mijwel et al. } \\
\text { (2019) }\end{array}$ & $\begin{array}{l}\text { Medicine and Science } \\
\text { in Sports and Exercise }\end{array}$ & $\begin{array}{l}\mathrm{N}=240 \\
\mathrm{GC}=80 \\
\mathrm{RT}-\mathrm{HIIT}=80 \\
\text { AT-HITT }=80 \\
18-70 \text { años }\end{array}$ & $\begin{array}{l}\text { Determinar los efectos del ejercicio en las tasas de finalización de la } \\
\text { quimioterapia y la hospitalización en personas con cáncer de mama que } \\
\text { se encuentran en quimioterapia. }\end{array}$ & $=16$ semanas & $\begin{array}{l}\text { El entrenamiento de resistencia e intervalos de alta intensidad durante la } \\
\text { quimioterapia pueden tener implicaciones significativas para el costo de la } \\
\text { atención del cáncer debido a las tasas reducidas de hospitalización, pero no } \\
\text { tuvo ningún efecto sobre las tasas de finalización del tratamiento con } \\
\text { quimioterapia. }\end{array}$ \\
\hline $\begin{array}{l}\text { Mijwel et al. } \\
\text { (2018) }\end{array}$ & The FASEB Journal & $\begin{array}{l}\mathrm{N}=23 \\
\text { GC }=10 \\
\text { RT-HIIT }=6 \\
\text { AT-HIIT }=7 \\
\text { GC }=51,0 \pm 13,1 \text { años } \\
\text { RT-HIIT }=54,3 \pm 11,0 \text { años } \\
\text { AT-HIIT }=51,5 \pm 7,0 \text { años }\end{array}$ & $\begin{array}{l}\text { Comparar los efectos entre diferentes programas de ejercicio, } \\
\text { diferentes al habitual, sobre la morfologia del músculo esquelético y } \\
\text { los marcadores mitocondriales en personas con cáncer de mama que se } \\
\text { encuentran en quimioterapia. }\end{array}$ & 16 semanas & $\begin{array}{l}\text { Las adaptaciones del entrenamiento a nivel musculoesquelético pueden } \\
\text { ocurrir junto con la quimioterapia. } \\
\text { Es importante implementar estos programas durante la quimioterapia para } \\
\text { prevenir los efectos secundarios negativos de la quimioterapia, preservando } \\
\text { la masa y la función del músculo. }\end{array}$ \\
\hline $\begin{array}{l}\text { Mijwel et al. } \\
(2019)\end{array}$ & $\begin{array}{l}\text { Journal of Cancer } \\
\text { Survivorship }\end{array}$ & $\begin{array}{l}\mathrm{N}=206 \\
\mathrm{GC}=52 \\
\text { RT-HIIT }=62 \\
\text { AT-HIIT }=59 \\
18-70 \text { años }\end{array}$ & $\begin{array}{l}\text { Examinar los efectos, } 12 \text { meses después, de dos intervenciones de } \\
\text { ejercicio sobre los resultados fisiológicos relacionados con la salud en } \\
\text { personas con cáncer de mama que se encuentran en quimioterapia. }\end{array}$ & 16 semanas & $\begin{array}{l}\text { Estos hallazgos enfatizan los efectos beneficiosos del ejercicio supervisado de } \\
\text { alta intensidad durante la quimioterapia para mejorar la salud y reducir los } \\
\text { costos sociales asociados con la baja por enfermedad, prolongada para } \\
\text { pacientes con cáncer de mama a varios meses después de la quimioterapia. }\end{array}$ \\
\hline $\begin{array}{l}\text { Northey et al. } \\
\text { (2019) }\end{array}$ & $\begin{array}{l}\text { Journal of Science and } \\
\text { Medicine in Sport }\end{array}$ & $\begin{array}{l}\mathrm{N}=17 \\
\mathrm{GC}=6 \\
\mathrm{GH}=6 \\
\mathrm{GIM}=5 \\
62,9 \pm 7,8 \text { años }\end{array}$ & $\begin{array}{l}\text { Efectos de dos intervenciones de ejercicio sobre la función cognitiva en } \\
\text { mujeres que han sobrevivido al cáncer de mama. }\end{array}$ & 12 semanas & $\begin{array}{l}\text { HIIT puede ser una intervención de ejercicio efectiva para mejorar el } \\
\text { rendimiento cognitivo, la función cerebrovascular y la capacidad aeróbica en } \\
\text { las sobrevivientes al cáncer de mama. }\end{array}$ \\
\hline $\begin{array}{l}\text { Schlüter et al. } \\
\text { (2019) }\end{array}$ & $\begin{array}{l}\text { Medicine \& Science in } \\
\text { Sports \& Exercise }\end{array}$ & $\begin{array}{l}\mathrm{N}=40 \\
20 \text { cáncer de mama } \\
62.9 \pm 9.2 \text { años }\end{array}$ & $\begin{array}{l}\text { Examinar dos protocolos HIIT para determinar su seguridad, } \\
\text { viabilidad y respuestas agudas al ejercicio en mujeres que han } \\
\text { sobrevivido al cáncer de mama. }\end{array}$ & 1 sesión & $\begin{array}{l}\text { Ambos protocolos son seguros, factibles y agradables. Aunque el programa } 4 \\
\mathrm{x} 4 \text { provocó un mayor gasto energético y una mayor tensión } \\
\text { cardiocirculatoria y metabólica. }\end{array}$ \\
\hline $\begin{array}{l}\text { Schmitt et al. } \\
\text { (2016) }\end{array}$ & Physiological Reports & $\begin{array}{l}\mathrm{N}=28 \\
\mathrm{GIM}=14 \\
\mathrm{GH}=14 \\
\mathrm{GIM}=54 \pm 9 \text { años } \\
\mathrm{GH}=53 \pm 8 \text { años }\end{array}$ & $\begin{array}{l}\text { Efectos de un programa HIIT sobre variables de aptitud aeróbica, } \\
\text { composición corporal, fasto energético, fatiga y calidad de vida } \\
\text { respecto a un programa habitual en mujeres sobrevivientes de cáncer } \\
\text { de mama. }\end{array}$ & 3 semanas & $\begin{array}{l}\text { Es factible realizar una intervención multimodal con HIIT sin efectos } \\
\text { adversos para la salud. Producen una mayor economía laboral, calidad de } \\
\text { vida y menos fatiga, pero no altera la composición corporal o el gasto } \\
\text { energético. El HIIT es una estrategia muy eficiente por su efecto en poco } \\
\text { tiempo. }\end{array}$ \\
\hline $\begin{array}{l}\text { Schulz et al. } \\
(2018)\end{array}$ & $\begin{array}{l}\text { Disability and } \\
\text { Rehabilitation }\end{array}$ & $\begin{array}{l}\mathrm{N}=26 \\
\mathrm{GH}=15 \\
\mathrm{GC}=11 \\
\mathrm{GH}=51.9 \pm 9.8 \text { años } \\
\mathrm{GC}=56.9 \pm 7.0 \text { años }\end{array}$ & $\begin{array}{l}\text { Evaluar la viabilidad de una intervención de entrenamiento combinado } \\
\text { de resistencia y fuerza con HIIT en mujeres con cáncer de mama. }\end{array}$ & 6 semanas & $\begin{array}{l}\text { Mejora a la capacidad aeróbica a corto plazo, manteniendo la capacidad de } \\
\text { desempeño y calidad de vida y una mejora de la resistencia, fuerza, calidad } \\
\text { de vida y seguridad del método. }\end{array}$ \\
\hline $\begin{array}{l}\text { Wang et al. } \\
(2019)\end{array}$ & BMC Cancer & $\begin{array}{l}\mathrm{N}=30 \\
\mathrm{GC}=15 \\
\mathrm{GH}=15 \\
46.9 \pm 9.8 \text { años }\end{array}$ & $\begin{array}{l}\text { Examinar los efectos del HIIT sobre la composición corporal en } \\
\text { mujeres con cáncer de mama sometidas a antraciclinas. }\end{array}$ & 8 semanas & $\begin{array}{l}\text { Una intervención HIIT de } 8 \text { semanas no mejoró la composición corporal en } \\
\text { pacientes obesas con cáncer de mama sometidas a terapia con antraciclina. }\end{array}$ \\
\hline $\begin{array}{l}\text { Lee et al. } \\
(2020)\end{array}$ & $\begin{array}{l}\text { Cancer epidemiology } \\
\text { biomarkers \& } \\
\text { prevention }\end{array}$ & $\begin{array}{l}\mathrm{N}=22 \\
\mathrm{GC}=11 \\
\mathrm{GH}=11 \\
48,9 \pm 6,2 \text { años }\end{array}$ & $\begin{array}{l}\text { Determinar si una intervención HIIT en mujeres con cáncer de mama } \\
\text { tratadas con antraciclinas es una estrategia factible. }\end{array}$ & 8 semanas & $\begin{array}{l}\text { Una intervención HIIT indujo a al mantenimiento del VO2max mientras } \\
\text { que el GC disminuyo su VO2max. Resulta segura y factible este tipo de } \\
\text { intervención en esta población. }\end{array}$ \\
\hline $\begin{array}{l}\text { Toohey et al. } \\
(2020)\end{array}$ & BMC Cancer & $\begin{array}{l}\mathrm{N}=17 \\
\mathrm{GC}=6 \\
\mathrm{GH}=6 \\
\mathrm{GIM}=5 \\
62 \pm 8 \text { años }\end{array}$ & $\begin{array}{l}\text { Explorar el impacto del HIIT en la aptitud aeróbica, regulación } \\
\text { cardiaca, biomarcadores salivales de estrés, inmunidad y marcadores de } \\
\text { mayor riesgo de enfermedades cardiovasculares en mujeres } \\
\text { supervivientes de cáncer de mama. }\end{array}$ & 12 semanas & $\begin{array}{l}\text { Doce semanas de intervención con HIIT mejora la aptitud cardiovascular, la } \\
\text { respuesta del sistema nervioso simpático y la regulación cardiaca. Por lo } \\
\text { tanto, puede reducir el riesgo de enfermedades cardiovasculares. }\end{array}$ \\
\hline $\begin{array}{l}\text { Coletta et al. } \\
(2021)\end{array}$ & $\begin{array}{l}\text { Breast Cancer research } \\
\text { and treatment }\end{array}$ & $\mathrm{N}=33$ & $\begin{array}{l}\text { Obtener la protección inmunológica del entrenamiento HIIT respecto } \\
\text { a la intensidad moderada en mujeres con alto riesgo de cáncer de } \\
\text { mama. }\end{array}$ & 12 semanas & $\begin{array}{l}\text { No hay diferencias significativas en la protección inmunológica, excepto en } \\
\text { mujeres con niveles cardiorrespiratorios bajos. }\end{array}$ \\
\hline $\begin{array}{l}\text { Pagola et al. } \\
(2020)\end{array}$ & $\begin{array}{l}\text { International journal c } \\
\text { sports medicine }\end{array}$ & $\begin{array}{l}\mathrm{N}=23 \\
\mathrm{GH}=13 \\
\mathrm{GIM}=10 \\
50 \pm 8 \text { años }\end{array}$ & $\begin{array}{l}\text { Obtención de mejores significativas de una intervención de } \\
\text { entrenamiento concurrente con HIIT respecto a una intensidad } \\
\text { moderada en personas con cáncer de mama. }\end{array}$ & 16 semanas & $\begin{array}{l}\text { No hay diferencias significativas entre ambos grupos, aunque los dos aportan } \\
\text { fuerza en la musculatura de las extremidades inferiores, mejor percepción } \\
\text { de la fatiga y mejor calidad de vida. }\end{array}$ \\
\hline
\end{tabular}




\begin{tabular}{|c|c|c|c|c|c|}
\hline $\begin{array}{l}\text { Schulz et al. } \\
\text { (2021) }\end{array}$ & $\begin{array}{l}\text { Disability and } \\
\text { rehabilitation }\end{array}$ & $\begin{array}{l}\mathrm{N}=19 \\
\mathrm{GC}=9 \\
\mathrm{GH}=10 \\
\mathrm{GC}=58,8 \pm 6,6 \text { años } \\
\mathrm{GH}=58,7 \pm 8,4 \text { años }\end{array}$ & $\begin{array}{l}\text { Viabilidad de la combinación de entrenamiento de resistencia y fuerza } \\
\text { con HIIT en mujeres con cáncer de mama no metastático para su } \\
\text { mejora psicológica y rendimiento.Y el mantenimiento de los efectos a } \\
\text { las } 12 \text { meses y } 24 \text { meses. }\end{array}$ & 6 semanas & $\begin{array}{l}\text { Mejora de del bienestar y rendimiento psicológico. Pero existe un retroceso } \\
\text { rápido de la capacidad aeróbica, el nivel de actividad y el estado } \\
\text { proinflamatorio a partir de los } 12 \text { meses. }\end{array}$ \\
\hline $\begin{array}{l}\text { Chung et al. } \\
\text { (2021) }\end{array}$ & $\begin{array}{l}\text { Annals of physical and } \\
\text { rehabilitation medicine }\end{array}$ & $\mathrm{N}=32$ & $\begin{array}{l}\text { Efecto del entrenamiento de intensidad moderada a alta en mujeres } \\
\text { con cáncer de mama en quimioterapia respecto a la función cardiaca. }\end{array}$ & 12 semanas & $\begin{array}{l}\text { El ejercicio a intensidades de moderada a alta puede prevenir el detrimento } \\
\text { de la función cardiaca. }\end{array}$ \\
\hline $\begin{array}{l}\text { Bell et al. } \\
(2021)\end{array}$ & $\begin{array}{l}\text { Supportive care in } \\
\text { cancer }\end{array}$ & $\begin{array}{l}N=20 \\
35-60 \text { años }\end{array}$ & $\begin{array}{l}\text { Determinar si el HIIT causa mayor VO2max que el ejercicio de } \\
\text { intensidad moderada en supervivientes de cáncer de mama entrenados. }\end{array}$ & 12 semanas & $\begin{array}{l}\text { Ambos grupos tuvieron mejora en el VO2 pico, pero no hubo diferencias } \\
\text { significativas entre ambos. }\end{array}$ \\
\hline $\begin{array}{l}\text { Hiensch et al. } \\
\text { (2021) }\end{array}$ & $\begin{array}{l}\text { Medicine and science in } \\
\text { sports and exercise }\end{array}$ & $\begin{array}{l}\mathrm{N}=240 \\
\mathrm{GC}=81 \\
\mathrm{RT}-\mathrm{HIIT}=79 \\
\text { AT-HIIT }=80 \\
18-70 \text { años }\end{array}$ & $\begin{array}{l}\text { Efectos beneficiosos del HIIT en la fatiga durante el tratamiento con } \\
\text { quimioterapia. }\end{array}$ & 16 semanas & $\begin{array}{l}\text { El ejercicio de resistencia e intervalos de alta intensidad produce niveles más } \\
\text { bajos de fatiga post intervención. }\end{array}$ \\
\hline $\begin{array}{l}\text { Ansund et al. } \\
(2021)\end{array}$ & Cardio-oncology & $\begin{array}{l}N=88 \\
18-70 \text { años }\end{array}$ & $\begin{array}{l}\text { Determinar el efecto cardioprotector que puede inducir el ejercicio } \\
\text { físico en una intervención con quimioterapia. }\end{array}$ & 16 semanas & $\begin{array}{l}\text { Niveles más bajos de biomarcadores y mayor VO2 pico mediante el } \\
\text { entrenamiento de resistencia junto al HIIT. }\end{array}$ \\
\hline $\begin{array}{l}\text { Lee et al. } \\
\text { (2021) }\end{array}$ & $\begin{array}{l}\text { Supportive Care in } \\
\text { Cancer }\end{array}$ & $\begin{array}{l}\mathrm{N}=30 \\
\mathrm{GC}=15 \\
\mathrm{GH}=15 \\
=18 \text { años }\end{array}$ & $\begin{array}{l}\text { Efectos en la función física en pacientes con cáncer de mama en } \\
\text { quimioterapia con antraciclinas mediante una intervención HIIT. }\end{array}$ & 8 semanas & $\begin{array}{l}\text { El HIIT puede mejorar la función física y la calidad de vida de las mujeres } \\
\text { con cáncer de mama en quimioterapia mediante antraciclinas. }\end{array}$ \\
\hline $\begin{array}{l}\text { Baldelli et al. } \\
\text { (2021) }\end{array}$ & $\begin{array}{l}\text { Clinical and } \\
\text { Translational Oncology }\end{array}$ & $\begin{array}{l}\mathrm{N}=30 \\
12 \text { mujeres, } 21,0 \pm 0,8 \text { años } \\
18 \text { hombres, } 21,0 \pm 1,4 \text { años }\end{array}$ & $\begin{array}{l}\text { Evaluar los efectos del HIIT sobre la proliferación de tumores } \\
\text { cancerígenos de mama y próstata. }\end{array}$ & 9 semanas & $\begin{array}{l}\text { Las sesiones de resistencia de alta intensidad promueven el control de la } \\
\text { progresión tumoral, por lo que puede prevenir el cáncer terciario. }\end{array}$ \\
\hline $\begin{array}{l}\text { Wiggenraad } \\
\text { et al. (2020) }\end{array}$ & $\begin{array}{l}\text { Integrative cancer } \\
\text { therapies }\end{array}$ & $\begin{array}{l}\mathrm{N}=240 \\
18-70 \text { años }\end{array}$ & $\begin{array}{l}\text { Evaluar longitudinalmente los cambios de los síntomas en pacientes } \\
\text { con cáncer de mama. }\end{array}$ & 16 semanas & $\begin{array}{l}\text { Efecto significativo del HIIT en los síntomas emocionales tras la } \\
\text { intervención y a largo plazo en pacientes con cáncer de mama tratados con } \\
\text { quimioterapia. }\end{array}$ \\
\hline $\begin{array}{l}\text { Bizjak et al. } \\
\text { (2020) }\end{array}$ & Cancers & $\begin{array}{l}\mathrm{N}=15 \\
\mathrm{GC}=6 \\
\mathrm{GH}=9\end{array}$ & $\begin{array}{l}\text { Papel de HIIT en la regulación del metabolismo del músculo } \\
\text { esquelético, respuesta al estrés oxidativo e influencia en la mutación. }\end{array}$ & 6 semanas & $\begin{array}{l}\text { El entrenamiento combinado de fuerza y resistencia a intervalos de alta } \\
\text { intensidad pueden reducir las mutaciones en personas propensas al cáncer. } \\
\text { Además, no se observó una respuesta proinflamatoria. }\end{array}$ \\
\hline
\end{tabular}

puede prevenir (Mijwel, Backman, Bolam, Jervaeus, et al., 2018). Incluso con una intervención multimodal combinando intensidad alta y baja (Adamsen et al., 2009) o con entrenamiento concurrente a intensidades moderadas o intensidades altas (Pagola et al., 2020).

Si el HIIT se combina con la nutrición se reduce esta fatiga relacionada con el cáncer en mujeres supervivientes de este (Schmitt et al., 2016).

En personas con cáncer de mama que están siendo tratadas con quimioterapia se ve una leve mejora de la composición corporal $(\mathrm{P}=0,24-0,28)$, pero menos que con otros métodos de entrenamiento (Bolam et al., 2019; Mijwel, Backman, Bolam, Olofsson, et al., 2018). Aunque no existen demasiados cambios significativos en personas obesas (Wang et al., 2019), en personas con alto riesgo de padecer cáncer de mama (Coletta et al., 2019), ni en personas supervivientes de cáncer (Schmitt et al., 2016b).

Por lo tanto, no hay un aumento de gasto calórico superior en personas supervivientes (Schmitt et al., 2016). Aunque si existen protocolos que tienen mayor gasto calórico que otros como por ejemplo 4 series de 4 minutos al 85\% - 95\% de la frecuencia cardiaca respecto a 10 series de 1 minuto a potencia máxima (Schlüter et al., 2019).

\section{Aspectos fisiológicos}

El ejercicio va a mejorar los efectos perjudiciales de la quimioterapia en el músculo esquelético (Mijwel, Cardinale, Norrbom, Chapman, et al., 2018), además se observó una mejora en la función endotelial vascular, un marcador clínico para pronosticar el desarrollo y/o resultado de enfermedad cardiovascular (Lee, Kang, Mack, Mortimer, Sattler, Salem, Lu, et al., 2019)y va a tener un impacto positivo en la resistencia y en la disminución de citoquinas inflamatorias con la consiguiente mejora en la respuesta inmune en pacientes con cáncer de mama (S. Alizadeh et al., 2019). Aunque, en el estudio de Coletta et al. (2021) podemos observar este aumento de la inmunidad se puede dar tanto con ejercicios a intensidades altas como a intensidades moderadas, excepto en mujeres con capacidad cardiorrespiratoria baja a las que le beneficia el HIIT.

No se han dado respuesta proinflamatorias tras el HIIT, pero si la reducción de las posibles mutaciones en personas propensas al cáncer (Bizjak et al., 2020b).

\section{Calidad de vida}

Existe controversia sobre el rendimiento cognitivo, ya que en personas con cáncer de mama en tratamiento de quimioterapia se ve una leve mejoría (Bolam et al., 2019), pero en otros estudios no hay una mejora respecto a la cognición (Mijwel, Backman, Bolam, Olofsson, et al., 2018). Aunque podemos observar que cuando se da un entrenamiento concurrente en el que se utiliza el HIIT, existe una mejora significativa en el bienestar y el rendimiento cognitivo(Schulz et al., 2021). En personas supervivientes del cáncer de mama tienen una respuesta positiva muy significativa respecto al rendimiento cognitivo y la función cerebrovascular, específicamente en la memoria episódica, memoria de trabajo y función ejecutiva (Northey et al., 2019), aunque se trata de un estudio piloto.

Con este tipo de intervención, la función emocional aumenta (Mijwel, Backman, Bolam, Olofsson, et al., 2018), incluso a largo plazo (Wiggenraad et al., 2020), y se reduce la fatiga emocional que produce esta enfermedad (Adamsen et al., 2009; Mijwel, Jervaeus, et al., 
2019). También se produce una reducción del estrés (Toohey et al., 2020) y la depresión en algunas personas supervivientes del cáncer de mama (Midtgaard et al., 2013). Atendiendo a la mejora de estas variables, es posible que la salud mental se vea afectada positivamente (Adamsen et al., 2009; Bolam et al., 2019; Midtgaard et al., 2013).

Se produce una mejora de la sintomatología de los pacientes tratados con quimioterapia (Bolam et al., 2019; Mijwel, Backman, Bolam, Jervaeus, et al., 2018). Se previenen los efectos secundarios negativos (Mijwel, Cardinale, Norrbom, Chapman, et al., 2018).

Además, existe una reducción de la sensibilidad al dolor modificando los umbrales de presión-dolor, especialmente en trapecio y glúteo (Mijwel, Backman, Bolam, Olofsson, et al., 2018).

Por lo tanto, teniendo en cuenta todos estas variables comentadas anteriormente, podemos observar una mejora en la calidad de vida de las personas con cáncer de mama (Lee et al., 2021; Pagola et al., 2020; Schmitt et al., 2016a; Schulz et al., 2018).

\section{Seguridad y factibilidad}

Este método es seguro y factible en personas que tienen un alto riesgo genético de cáncer de mama (Coletta et al., 2019), en pacientes con cáncer de mama durante el tratamiento de quimioterapia (Lee et al., 2020; Schulz et al., 2018) y en supervivientes de cáncer de mama (Adamsen et al., 2009; Schlüter et al., 2019) a pesar de que la intensidad sea vigorosa y tengan alta probabilidad de padecer, padezcan o hayan padecido cáncer.

\section{Tasa de finalización, hospitalización y costos}

El HIIT en pacientes con cáncer de mama con quimioterapia repercute en las tasas de hospitalización reduciéndose, pero no disminuye el tiempo de tratamiento con quimioterapia (Mijwel, Bolam, et al., 2019; Mijwel et al., 2020). Esto implica la reducción de costos sanitarios asociados a este tipo de cáncer (Mijwel, Bolam, et al., 2019), a complicaciones relacionadas con el tratamiento (Mijwel et al., 2020) y también se reducen costos sociales asociados a bajas prolongadas tras el paso por quimioterapia, ya que estas disminuyen (Mijwel, Jervaeus, et al., 2019). Además, este tipo de intervención promueve el control del progreso tumoral pudiendo prevenir ciertos tipos de cáncer, pudiendo reducir el costo que supondría un futuro tratamiento (Baldelli et al., 2021).

\section{Conclusión}

Basándonos en los hallazgos de la literatura científica analizada, podemos concluir que existen evidencias que señalan los beneficios del HIIT en personas con cáncer de mama.

El uso de este método durante el tratamiento en personas con cáncer de mama produce un aumento de la resistencia, fuerza muscular, rendimiento cognitivo y se reduce la fatiga física y emocional, al igual que los efectos secundarios y el dolor provocado por la enfermedad. En personas supervivientes del cáncer de mama se da una mejora de la resistencia y una disminución de la fatiga, síntomas y efectos secundarios.

Para todos estos casos existe una gran seguridad, fiabilidad y factibilidad del método, reduciendo costos sanitarios.

En resumen, la literatura consultada muestra la viabilidad y la eficacia de este programa de intervención basado en HIIT para la mejora de las capacidades física, aspectos fisiológicos, calidad de vida, seguridad y factibilidad del programa y tasas de finalización, hospitalización y costos. Esta revisión trata de aportar información útil y relevante para los profesionales del ámbito médico, fisioterapeutas y profesionales en el deporte en cuanto a la utilización de este método para este colectivo. Los datos obtenidos podrían ser utilizados para la elaboración de guías de práctica clínica utilizadas como complemento en el tratamiento de estos pacientes. Futuros estudios, deberán determinar el potencial del HIIT en personas con cáncer de mama, aumentando el tamaño muestral y dividiéndola por edades y realizando un protocolo como base de este tipo de intervención.

\section{Referencias}

Adamsen, L., Quist, M., Andersen, C., Moller,T., Herrstedt, J., Kronborg, D., Baadsgaard, M.T., Vistisen, K., Midtgaard, J., Christiansen, B., Stage, M., Kronborg, M.T., \& Rorth, M. (2009). Effect of a multimodal high intensity exercise intervention in cancer patients undergoing chemotherapy: Randomised controlled trial. Bmj-British Medical Journal, 339, b3410. https://doi.org/10.1136/bmj.b3410

Alizadeh, A. M., Isanejad, A., Sadighi, S., Mardani, M., kalaghchi, B., \& Hassan, Z. M. (2019). High-intensity interval training can modulate the systemic inflammation and HSP70 in the breast cancer: A randomized control trial. Journal of Cancer Research and Clinical Oncology, 145(10), 2583-2593. https://doi.org/10.1007/s00432-01902996-y 
Alizadeh, S., Isanejad, A., Sadighi, S., Khalighfard, S., \& Alizadeh, A. M. (2019). Effect of a high-intensity interval training on serum microRNA levels in women with breast cancer undergoing hormone therapy. A single-blind randomized trial. Annals of Physical and Rehabilitation Medicine, 62(5), 329-335.

j.rehab.2019.07.001

Ansund, J., Mijwel, S., Bolam, K. A., Altena, R., Wengström, Y., Rullman, E., \& Rundqvist, H. (2021). High intensity exercise during breast cancer chemotherapy_Effects on long-term myocardial damage and physical capacityData from the OptiTrain RCT. Cardio-Oncology, 7(1), 7. https: / / doi.org/10.1186/s40959-021-00091-1

Baldelli, G., De Santi, M., Gervasi, M., Annibalini, G., Sisti, D., Højman, P., Sestili, P., Stocchi, V., Barbieri, E., \& Brandi, G. (2021). The effects of human sera conditioned by highintensity exercise sessions and training on the tumorigenic potential of cancer cells. Clinical andTranslational Oncology, 23(1), 22-34. https://doi.org/10.1007/s12094-02002388-6

Bell, R. A., Baldi, J. C., \& Jones, L. M. (2021). Additional cardiovascular fitness when progressing from moderateto high-intensity exercise training in previously trained breast cancer survivors. Supportive Care in Cancer. https: / / doi.org/10.1007/s00520-021-06259-w

Bizjak, D. A., Schulz, S.V.W., Schumann, U., Otto, S., Kirsten, J., Ebner, F., Leinert, E., Huober, J., Janni, W., \& Steinacker, J. M. (2020a). Beneficial Molecular Adaptations in BRCAMutation Carriers by Combined HIT/HIRT Intervention: Results from a Pilot Study. Cancers, 12(6), 1526. https: / / doi.org/10.3390/cancers12061526

Bizjak, D.A., Schulz, S.V.W., Schumann, U., Otto, S., Kirsten, J., Ebner, F., Leinert, E., Huober, J., Janni, W., \& Steinacker, J. M. (2020b). Beneficial Molecular Adaptations In BRCAMutation Carriers By Combined HIT/HIRT Intervention: Results From A Pilot Study. Cancers, 12(6). https:/ / doi.org/10.3390/cancers12061526

Blackwell, J. E. M., Doleman, B., Boereboom, C. L., Morton, A., Williams, S., Atherton, P., Smith, K., Williams, J. P., Phillips, B. E., \& Lund, J. N. (2020). High-intensity interval training produces a significant improvement in fitness in less than 31 days before surgery for urological cancer: A randomised control trial. Prostate Cancer and Prostatic Diseases. https://doi.org/10.1038/s41391-020-0219. 1

Bolam, K. A., Mijwel, S., Rundqvist, H., \& Wengström, Y. (2019). Two-year follow-up of the OptiTrain randomised controlled exercise trial. Breast Cancer Research and Treatment, 175(3), 637-648. https://doi.org/10.1007/ s10549-019-05204-0

Chung,W.-P.,Yang, H.-L., Hsu,Y.-T., Hung, C.-H., Liu, P.-Y., Liu, Y.-W., Chan, S.-H., \& Tsai, K.-L. (2021). Real-time exercise reduces impaired cardiac function in breast cancer patients undergoing chemotherapy: A randomized controlled trial. Annals of Physical and Rehabilitation Medi- cine, 3, 101485. https://doi.org/10.1016/ j.rehab.2021.101485

Coletta, A. M., Agha, N. H., Baker, F. L., Niemiro, G. M., Mylabathula, P. L., Brewster, A. M., Bevers, T. B., FuentesMattei, E., Basen-Engquist, K., Gilchrist, S. C., \& Simpson, R. J. (2021). The impact of high-intensity interval exercise training on NK-cell function and circulating myokines for breast cancer prevention among women at high risk for breast cancer. Breast Cancer Research and Treatment, 187(2), 407-416. https://doi.org/10.1007/s10549021-06111-z

Coletta, A. M., Brewster, A. M., Chen, M., Li,Y., Bevers, T. B., Basen-Engquist, K., \& Gilchrist, S. C. (2019). HighIntensity Interval Training Is Feasible in Women at High Risk for Breast Cancer: Medicine \& Science in Sports \& Exercise, 51(11), 2193-2200. https://doi.org/10.1249/ MSS.0000000000002048

D’Ascenzi, F., Anselmi, F., Fiorentini, C., Mannucci, R., Bonifazi, M., \& Mondillo, S. (2019). The benefits of exercise in cancer patients and the criteria for exercise prescription in cardio-oncology. European Journal of Preventive Cardiology, 204748731987490. https: / / doi.org/10.1177/2047487319874900

Dennett, A. M., Peiris, C. L., Shields, N., Prendergast, L.A., \& Taylor, N. F. (2016). Moderate-intensity exercise reduces fatigue and improves mobility in cancer survivors: A systematic review and meta-regression. Journal of Physiotherapy, 62(2), 68-82. https://doi.org/10.1016/ j.jphys.2016.02.012

Emberts, T., Porcari, J., Dobers-tein, S., Steffen, J., \& Foster, C. (2013). Exercise Intensity and Energy Expenditure of aTabataWorkout. Journal of Sports Science \& Medicine, 12(3), 612-613.

Foerster, B., Pozo, C., Abufaraj, M., Mari, A., Kimura, S., D’Andrea, D., John, H., \& Shariat, S. F. (2018). Association of Smoking Status With Recurrence, Metastasis, and Mortality Among PatientsWith Localized Prostate Cancer Undergoing Prostatectomy or Radiotherapy: A Systematic Review and Meta-analysis. JAMA Oncology, 4(7), 953-961. https: / / doi.org/10.1001/jamaoncol.2018.1071

Forouzanfar, M. H., Afshin, A., Alexander, L. T., Anderson, H. R., Bhutta, Z. A., Biryukov, S., Brauer, M., Burnett, R., Cercy, K., Charlson, F. J., Cohen, A. J., Dandona, L., Estep, K., Ferrari, A. J., Frostad, J. J., Fullman, N., Gething, P.W., Godwin,W.W., Griswold, M., ... Murray, C. J. L. (2016). Global, regional, and national comparative risk assessment of 79 behavioural, environmental and occupational, and metabolic risks or clusters of risks, 1990-2015: A systematic analysis for the Global Burden of Disease Study 2015. The Lancet, 388(10053), 1659-1724. https:// doi.org/10.1016/S0140-6736(16)31679-8

Fuller, J. T., Hartland, M. C., Maloney, L. T., \& Davison, K. (2018). Therapeutic effects of aerobic and resistance exercises for cancer survivors: A systematic review of meta-analyses of clinical trials. British Journal of Sports Me- 
dicine, 52(20), 1311-1311. https://doi.org/10.1136/ bjsports-2017-098285

García, A. M. de O., Manzanedo, J. G., \& González, J. G. P. (2019). Entrenamiento Interválico de Alta Intensidad (HIIT) como herramienta terapéutica en pacientes con Diabetes MellitusTipo 2: Una revisión narrativa. Retos, 36, 633-639. https:// doi.org/10.47197/ retos.v36i36.69762

Gibala, M. J., \& McGee, S. L. (2008). Metabolic Adaptations to Short-term High-Intensity Interval Training: A Little Pain for a Lot of Gain? Exercise and Sport Sciences Reviews, 36(2), 58-63. https://doi.org/10.1097/ JES.0b013e318168ec1f

Gomez Piqueras, P., \& Sanchez Gonzalez, M. (2019). High Intensity Interval Training (hiit) in Older Adults: A Systematic Review. Pensar En Movimiento-Revista De Ciencias Del EjercicioY La Salud, 17(1), UNSP e35494. https: / /doi.org/10.15517/pensarmov.v17i1.35494

Grisalez, A. A. D., Quiceno, C. A. M., Herrera, A. L. C., \& Corrales, J. L. H. (2021). Efecto de un programa de entrenamiento interválico aeróbico de alta intensidad en población escolar femenina con sobrepeso u obesidad (Effect of a high intensity aerobic interval training program on an overweight or obese female school population). Retos, 39, 453-458. https://doi.org/10.47197/ retos.v0i39.78200

Heinrich, K. M., Patel, P. M., O’Neal, J. L., \& Heinrich, B. S. (2014). High-intensity compared to moderate-intensity training for exercise initiation, enjoyment, adherence, and intentions: An intervention study. Bmc Public Health, 14, 789. https://doi.org/10.1186/1471-2458-14-789

Hiensch,A. E., Mijwel, S., Bargiela, D., Wengström,Y., May,A. M., \& Rundqvist, H. (2021). Inflammation Mediates Exercise Effects on Fatigue in Patients with Breast Cancer. Medicine \& Science in Sports \& Exercise, 53(3), 496-504. https: / / doi.org/10.1249/MSS.0000000000002490

Karlsen, T., Aamot, I.-L., Haykowsky, M., \& Rognmo, Ø. (2017). High Intensity Interval Training for Maximizing Health Outcomes. Progress in Cardiovascular Diseases, 60(1), 67-77. https://doi.org/10.1016/j.pcad.2017.03.006

Lee, K., Kang, I., Mack, W. J., Mortimer, J., Sattler, F., Salem, G., \& Dieli-Conwright, C. M. (2019). Feasibility of high intensity interval training in patients with breast Cancer undergoing anthracycline chemotherapy: A randomized pilot trial. BMC Cancer, 19(1), 653. https://doi.org/ 10.1186/s12885-019-5887-7

Lee, K., Kang, I., Mack, W. J., Mortimer, J., Sattler, F., Salem, G., Lu, J., \& Dieli-Conwright, C. M. (2019). Effects of high-intensity interval training on vascular endothelial function and vascular wall thickness in breast cancer patients receiving anthracycline-based chemotherapy: A randomized pilot study. Breast Cancer Research andTreatment, 177(2), 477-485. https://doi.org/10.1007/s10549019-05332-7

Lee, K., Norris, M. K., Wang, E., \& Dieli-Conwright, C. M.
(2021). Effect of high-intensity interval training on patientreported outcomes and physical function in women with breast cancer receiving anthracycline-based chemotherapy. Supportive Care in Cancer. https: / / doi.org / 10.1007/s00520-021-06294-7

Lee, K., Ortiz, K., Goytizolo, J., Serrano,T., \& Dieli-Conwright, C. M. (2020). Abstract D033: High-intensity interval training is feasible in Hispanic patients with breast cancer undergoing anthracycline chemotherapy. Cancer Epidemiology and Prevention Biomarkers, $29(6$ Supplement 2), D033.

Midtgaard, J., Christensen, J. F., Tolver,A., Jones, L.W., Uth, J., Rasmussen, B., Tang, L., Adamsen, L., \& Rorth, M. (2013). Efficacy of multimodal exercise-based rehabilitation on physical activity, cardiorespiratory fitness, and patientreported outcomes in cancer survivors: A randomized, controlled trial. Annals of Oncology, 24(9), 2267-2273. https: / /doi.org/10.1093/annonc/mdt185

Mijwel, S., Backman, M., Bolam, K.A., Jervaeus, A., Sundberg, C. J., Margolin, S., Browall, M., Rundqvist, H., \& Wengström, Y. (2018). Adding high-intensity interval training to conventional training modalities: Optimizing health-related outcomes during chemotherapy for breast cancer: the OptiTrain randomized controlled trial. Breast Cancer Research and Treatment, 168(1), 79-93. https:// doi.org/10.1007/s10549-017-4571-3

Mijwel, S., Backman, M., Bolam, K. A., Olofsson, E., Norrbom, J., Bergh, J., Sundberg, C. J., Wengström,Y., \& Rundqvist, H. (2018). Highly favorable physiological responses to concurrent resistance and high-intensity interval training during chemotherapy: The OptiTrain breast cancer trial. Breast Cancer Research and Treatment, 169(1), 93-103. https: / / doi.org/10.1007/s10549018-4663-8

Mijwel, S., Bolam, K.A., Gerrevall, J., Foukakis, T., Wengström, Y., \& Rundqvist, H. (2020). Effects of Exercise on Chemotherapy Completion and Hospitalization Rates:The OptiTrain Breast Cancer Trial. The Oncologist, 25(1), 2332. https://doi.org/10.1634/theoncologist.20190262

Mijwel, S., Bolam, K. A., Wengstrom, Y., \& Rundqvist, H. (2019). Effects Of Exercise During Chemotherapy On Hospitalization And Chemotherapy Completion: The OptiTrain Breast CancerTrial. Medicine and Science in Sports and Exercise, 51(6), 881-881. https://doi.org/10.1249/ 01.mss.0000563131.82247.a8

Mijwel, S., Cardinale, D. A., Norrbom, J., Chapman, M., Ivarsson, N., Wengström,Y., Sundberg, C. J., \& Rundqvist, H. (2018). Exercise training during chemotherapy preserves skeletal muscle fiber area, capillarization, and mitochondrial content in patients with breast cancer. The FASEB Journal, 32(10), 5495-5505. https://doi.org/ 10.1096/fj.201700968R

Mijwel, S., Jervaeus, A., Bolam, K. A., Norrbom, J., Bergh, J., Rundqvist, H., \& Wengström, Y. (2019). High-intensity 
exercise during chemotherapy induces beneficial effects 12 months into breast cancer survivorship. Journal of Cancer Survivorship, 13(2), 244-256. https: / / doi.org/10.1007/ s11764-019-00747-z

Neto, V. G. C., Rosário, J. A. do, Bodell, N., Araujo, G. da S., Telles, L. G. da S., Freitas, J. P. de, Colonna, M. J. C. M., Triani, F. da S., Silva, R. S. da, \& Monteiro, E. R. (2022). Blood pressure, heart rate, and rate pressure product behavior during interval and continuous aerobic exercise (Comportamiento de la presión arterial, frecuencia cardíaca y doble producto durante el ejercicio aeróbico a intervalos y continuo). Retos, 43, 579-585. https:// doi.org/10.47197/retos.v43i0.88363

Northey, J. M., Pumpa, K. L., Quinlan, C., Ikin, A., Toohey, K., Smee, D. J., \& Rattray, B. (2019). Cognition in breast cancer survivors: A pilot study of interval and continuous exercise. Journal of Science and Medicine in Sport, 22(5), 580-585. https: / / doi.org/10.1016/j.jsams.2018.11.026

Pagola, I., Morales, J. S., Alejo, L. B., Barcelo, O., Montil, M., Olivan, J., Alvarez-Bustos, A., Cantos, B., Maximiano, C., Hidalgo, F., Valenzuela, P. L., Fiuza-Luces, C., Lucia, A., \& Ruiz-Casado, A. (2020). Concurrent Exercise Interventions in Breast Cancer Survivors with Cancerrelated Fatigue. International Journal of Sports Medicine, 41(11), 790-797. https://doi.org/10.1055/a-11471513

Pérez, F. J. S., \& Chaves, G. A. C. (2015). Efectos del entrenamiento Tabata en la composición corporal del futbolista. Kronos: revista universitaria de la actividad física y el deporte, 14(1), 7.

Plummer, M., de Martel, C., Vignat, J., Ferlay, J., Bray, F., \& Franceschi, S. (2016). Global burden of cancers attributable to infections in 2012: A synthetic analysis. The Lancet. Global Health, 4(9), e609-616. https://doi.org/ 10.1016/S2214-109X(16)30143-7

Reljic, D., Lampe, D., Wolf, F., Zopf, Y., Herrmann, H. J., \& Fischer, J. (2019). Prevalence and predictors of dropout from high-intensity interval training in sedentary individuals: A meta-analysis. Scandinavian Journal of Medicine \& Science in Sports, 29(9), 1288-1304. https: / / doi.org/10.1111/sms. 13452

Schlüter, K., Schneider, J., Sprave, T., Wiskemann, J., \& Rosenberger, F. (2019). Feasibility of Two High-Intensity Interval Training Protocols in Cancer Survivors. Medicine and Science in Sports and Exercise, 51(12), 2443-2450. https: / / doi.org/10.1249/MSS.0000000000002081

Schmitt, J., Lindner, N., Reuss-Borst, M., Holmberg, H.-C., \& Sperlich, B. (2016a).A 3-week multimodal intervention involving high-intensity interval training in female cancer survivors: A randomized controlled trial. Physiological Reports, 4(3), e12693. https://doi.org/10.14814/ phy2.12693

Schmitt, J., Lindner, N., Reuss-Borst, M., Holmberg, H.-C., \& Sperlich, B. (2016b). A 3-week multimodal intervention involving high-intensity interval training in female cancer survivors: A randomized controlled trial. Physiological Reports, 4(3), e12693. https://doi.org/ 10.14814 /phy2.12693

Schulz, S. V. W., Laszlo, R., Otto, S., Prokopchuk, D., Schumann, U., Ebner, F., Huober, J., \& Steinacker, J. M. (2018). Feasibility and effects of a combined adjuvant highintensity interval/strength training in breast cancer patients: A single-center pilot study. Disability and Rehabilitation, 40(13), 1501-1508. https://doi.org/ 10.1080/09638288.2017.1300688

Schulz, S.V.W., Schumann, U., Otto, S., Kirsten, J., Treff, G., Janni,W., Huober, J., Leinert, E., Steinacker, J. M., \& Bizjak, D. A. (2021). Two-year follow-up after a six-week highintensity training intervention study with breast cancer patients: Physiological, psychological and immunological differences. Disability and Rehabilitation, O(0), 1-8. https: / /doi.org/10.1080/09638288.2021.1921861

Segovia, Y., \& Gutiérrez, D. (2020). Esfuerzo, Implicación y Condición Física percibida en un Programa HIIT en Educación Física. Modelo Educación Deportiva vs Metodología Tradicional (Perception of Exertion, involvement and physical fitness in a HIIT Program in Physical Education. Sport Edu. Retos, 38, 151-158. https:// doi.org/10.47197/retos.v38i38.73686

Toohey, K., Pumpa, K., McKune,A., Cooke, J., Welvaert, M., Northey, J., Quinlan, C., \& Semple, S. (2020).The impact of high-intensity interval training exercise on breast cancer survivors: A pilot study to explore fitness, cardiac regulation and biomarkers of the stress systems. BMC cancer, 20(1), 787. https://doi.org/10.1186/s12885-020-07295-1

Wang, E., Lee, K., \& Dieli-Conwright, C. M. (2019). Effects of High-Intensity Interval Training on Body Composition in Breast Cancer Patients Undergoing Anthracycline Chemotherapy. Medicine and Science in Sports and Exercise, 51(6), 879-879. https://doi.org/10.1249/ 01.mss.0000563126.07797.6d

Wiggenraad, F., Bolam, K. A., Mijwel, S., van der Wall, E., Wengström, Y., \& Altena, R. (2020). Long-Term Favorable Effects of Physical Exercise on Burdensome Symptoms in the OptiTrain Breast Cancer Randomized Controlled Trial. Integrative Cancer Therapies, 19, 1534735420905003. https://doi.org/10.1177/ 1534735420905003

Wilke, J., Kaiser, S., Niederer, D., Kalo, K., Engeroff, T., Morath, C., Vogt, L., \& Banzer,W. (2019). Effects of highintensity functional circuit training on motor function and sport motivation in healthy, inactive adults. Scandinavian Journal of Medicine \& Science in Sports, 29(1), 144-153. https: / / doi.org/10.1111/sms.13313

Zhang, X., Li,Y., \& Liu, D. (2019). Effects of exercise on the quality of life in breast cancer patients: A systematic review of randomized controlled trials. Supportive Care in Cancer, 27(1), 9-21. https://doi.org/10.1007/s00520-0184363-2 